\title{
Arginine methylation regulates mitochondrial gene expression in Trypanosoma brucei through multiple effector proteins
}

\author{
CHRISTOPHER C. GOULAH, MICHEL PELLETIER, and LAURIE K. READ \\ Department of Microbiology and Immunology and Witebsky Center for Microbial Pathogenesis and Immunology, \\ SUNY Buffalo School of Medicine, Buffalo, New York 14214, USA
}

\begin{abstract}
Arginine methylation is a post-translational modification that impacts gene expression in both the cytoplasm and nucleus. Here, we demonstrate that arginine methylation also affects mitochondrial gene expression in the protozoan parasite, Trypanosoma brucei. Down-regulation of the major trypanosome type I protein arginine methyltransferase, TbPRMT1, leads to destabilization of specific mitochondrial mRNAs. We provide evidence that some of these effects are mediated by the mitochondrial RNAbinding protein, RBP16, which we previously demonstrated affects both RNA editing and stability. TbPRMT1 catalyzes methylation of RBP16 in vitro. Further, MALDI-TOF-MS analysis of RBP16 isolated from TbPRMT1-depleted cells indicates that, in vivo, TbPRMT1 modifies two of the three known methylated arginine residues in RBP16. Expression of mutated, nonmethylatable RBP16 in $T$. brucei has a dominant negative effect, leading to destabilization of a subset of those mRNAs affected by TbPRMT1 depletion. Our results suggest that the specificity and multifunctional nature of RBP16 are due, at least in part, to the presence of differentially methylated forms of the protein. However, some effects of TbPRMT1 depletion on mitochondrial gene expression cannot be accounted for by RBP16 action. Thus, these data implicate additional, unknown methylproteins in mitochondrial gene regulation.
\end{abstract}

Keywords: arginine methylation; RNA binding; RNA stability; gene expression; trypanosome

\section{INTRODUCTION}

Protein arginine methylation is a widespread post-translational modification that increases the functional diversity of proteins. Arginine residues are modified through the transfer of a methyl group from S-adenosyl-L-methionine (AdoMet) to a nitrogen atom on the arginine side chain. This modification is catalyzed by two distinct classes of protein arginine methyltransferase (PRMT) enzymes. While both enzyme types generate monomethylarginine as an intermediate, type I enzymes catalyze the formation of asymmetric dimethylarginine residues and type II enzymes catalyze the formation of symmetric dimethylarginine residues (Bedford and Richard 2005). Arginine methylation often occurs in the context of RGG, RG, or RXR motifs of RNA-binding proteins (McBride and Silver 2001; Bedford and

Reprint requests to: Laurie K. Read, Department of Microbiology and Immunology, SUNY Buffalo School of Medicine, Buffalo, NY 14214, USA; e-mail: 1read@acsu.buffalo.edu; fax: (716) 829-2158.

Article published online ahead of print. Article and publication date are at http://www.rnajournal.org/cgi/doi/10.1261/rna.90106.
Richard 2005). The modification has been associated with a variety of cellular processes including signal transduction, subcellular localization, transcription, and RNA processing (McBride and Silver 2001; Cimato et al. 2002; Lukong and Richard 2004). The effects of methylation on these processes are primarily mediated through modulation of protein:protein interactions (Bedford et al. 2000; Côté et al. 2003; McBride et al. 2005). Although arginines are frequently found at protein: RNA interfaces (Gary and Clarke 1998), arginine methylation has only recently been reported to significantly affect protein:RNA interactions (Stetler et al. 2005). Thus, arginine methylation has the potential to control multiple aspects of gene expression through the regulation of both protein: protein and protein:RNA interactions. While the impact of arginine methylation on several nuclear and cytoplasmic gene regulatory processes has been reported, a role for arginine methylation in the regulation of mitochondrial gene expression has not been explored.

Trypanosoma brucei is a parasitic protozoan and the causative agent of African sleeping sickness. T. brucei contains a single large mitochondrion whose biologic 
functions are rapidly and drastically altered with environmental changes and life-cycle stages. Transcription of the mitochondrial genome is polycistronic (Read et al. 1992; Koslowsky and Yahampath 1997; Grams et al. 2000), and thus, regulation of mitochondrial gene expression primarily involves a complex coordination of post-transcriptional events. These include the processing of polycistronic transcripts by $3^{\prime}$ and $5^{\prime}$ cleavage, transcript maturation through polyadenylation and RNA editing, and RNA turnover (Bhat et al. 1992; Militello and Read 1999; Madison-Antenucci et al. 2002; Stuart and Panigrahi 2002; Simpson et al. 2003; Kao and Read 2005; Lukes et al. 2005). These events are controlled by strict regulatory mechanisms for which there are presumably many cis- and trans-acting factors that act to modulate transcript utilization and abundance (Ryan et al. 2003; Kao and Read 2005). RNA-binding proteins play a pivotal role in all aspects of cellular RNA processing and utilization (Dreyfuss et al. 2002), and several RNA-binding proteins have been described in T. brucei mitochondria, including TBRGG1 (Vanhamme et al. 1998), REAP-1 (Madison-Antenucci and Hajduk 2001), RBP16 (Pelletier and Read 2003), and MRP1/2 (Vondruskova et al. 2005). However, the mechanisms by which these proteins act are generally not well understood.

RBP16 is a Y-box family RNA-binding protein, which was shown by RNA interference (RNAi) studies to be involved in regulating mitochondrial RNA editing and stability in T. brucei (Pelletier and Read 2003). At its C terminus, RBP16 contains an RG-rich RNA-binding region (Miller and Read 2003). Previous studies have also demonstrated that arginine residues within the RBP16 C terminus are substrates for arginine methylation in vivo and that differentially methylated forms of the protein are present in mitochondria (Pelletier et al. 2001). Arginine methylation has been widely reported to affect the function of RNA-binding proteins including SAM68 (Côté et al. 2003) and yeast Npl3 (McBride et al. 2005). Thus, we hypothesized that differential arginine methylation of RBP16 might be important in mediating the diverse gene regulatory functions of this mitochondrial protein.

In this study, we examine the role of arginine methylation in trypanosome mitochondrial gene expression by monitoring RNA levels in cells down-regulated for the major trypanosome type I PRMT (TbPRMT1) by RNAi. We show that several mitochondrial RNAs NADH dehydrogenase subunit 4 (ND4), cytochrome oxidase I (COI), and both edited and unedited (COII) are specifically destabilized in these cells. We also demonstrate that RBP16 serves as a TbPRMT1 substrate both in vivo and in vitro, and we identify a select subset of arginine residues within RBP16 whose methylation is catalyzed by TbPRMT1 in vivo. To further examine the role of RBP16 as an effector of arginine methylation action in mitochondria, we overexpressed a nonmethylatable form of RBP16 and determined whether it had dominant negative consequences for RNA editing and/or stability. These studies reveal that RBP16 methylation is important in the stabilization of the neveredited ND4 RNA as well as the stabilization of both edited and unedited apocytochrome $\mathrm{b}(\mathrm{CYb})$ and COIII RNAs. Together, these data strongly suggest that specific RNA stabilization functions of RBP16 require TbPRMT1-catalyzed methylation. In addition, the dramatic effect on COII RNA levels in TbPRMT1 knock-down cells cannot be accounted for by RBP16 action, thereby implicating additional methylproteins in mitochondrial gene regulation. Overall, our data demonstrate that the methylation of effector proteins, including RBP16, by TbPRMT1 promotes the stabilization of multiple mitochondrial transcripts in T. brucei. These results constitute the first report of a role for arginine methylation in regulating mitochondrial gene expression.

\section{RESULTS}

\section{TbPRMT1 depletion reveals a role for arginine methylation in mitochondrial gene regulation}

TbPRMT1 is the trypanosome homolog of the mammalian PRMT1 and yeast HMT1 enzymes, and constitutes the major type I PRMT in T. brucei (Pelletier et al. 2005). We previously described the creation and initial characterization of a clonal procyclic form TbPRMT1 RNAi cell line (Pelletier et al. 2005). In the previous studies, we demonstrated that although growth was unaffected, total cellular asymmetric dimethylarginine was dramatically reduced in these cells on day 4 following tet induction of RNAi. To determine whether protein arginine methylation has an impact on mitochondrial gene expression in trypanosomes, we first examined mitochondrial RNA metabolism following tet-induced depletion of TbPRMT1 (Pelletier et al. 2005). Total RNA was isolated from TbPRMT1 RNAi cells either induced for $4 \mathrm{~d}$ in the presence of $2.5 \mu \mathrm{g} / \mathrm{mL}$ tet, or left uninduced prior to RNA isolation. Northern blot analysis confirmed that TbPRMT1 mRNA levels were reduced $>95 \%$ following tet induction (data not shown). Mitochondrial RNAs were quantified by poisoned primer extension (Pelletier and Read 2003). We analyzed seven mitochondrial RNAs representing three different classes of RNA editing-COIII and ATPase subunit 6 (A6), which are pan edited throughout their sequences; CYb and COII, which are edited within a small region; and COI, ND4, and maxicircle-unidentified reading frame 1 (MURF1), which are never edited. The RNAs examined include those whose expression is affected by down-regulation of the RNAbinding protein, RBP16 (CYb, COI, and ND4 RNAs), which is the only reported methylprotein in T. brucei (Pelletier et al. 2001). In these experiments, TbPRMT1 depletion had no effect on the levels of either edited or unedited A6 or COIII, indicating that TbPRMT1 does 

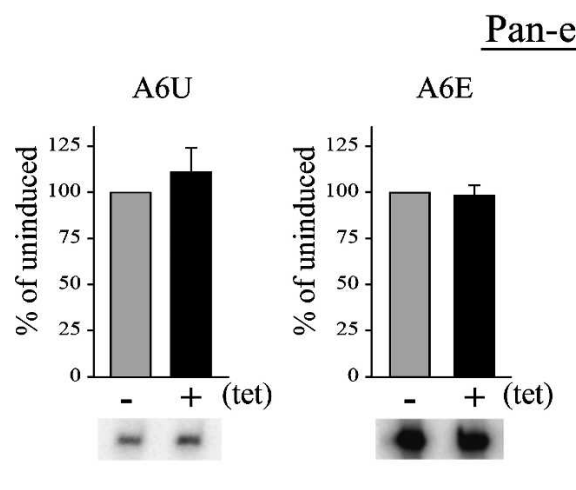

$\underline{\text { Pan-edited }}$
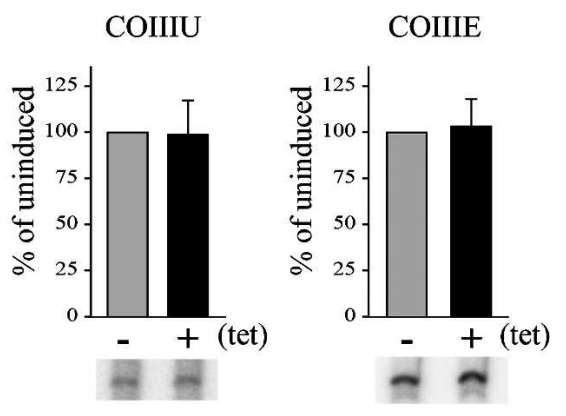

Minimally edited
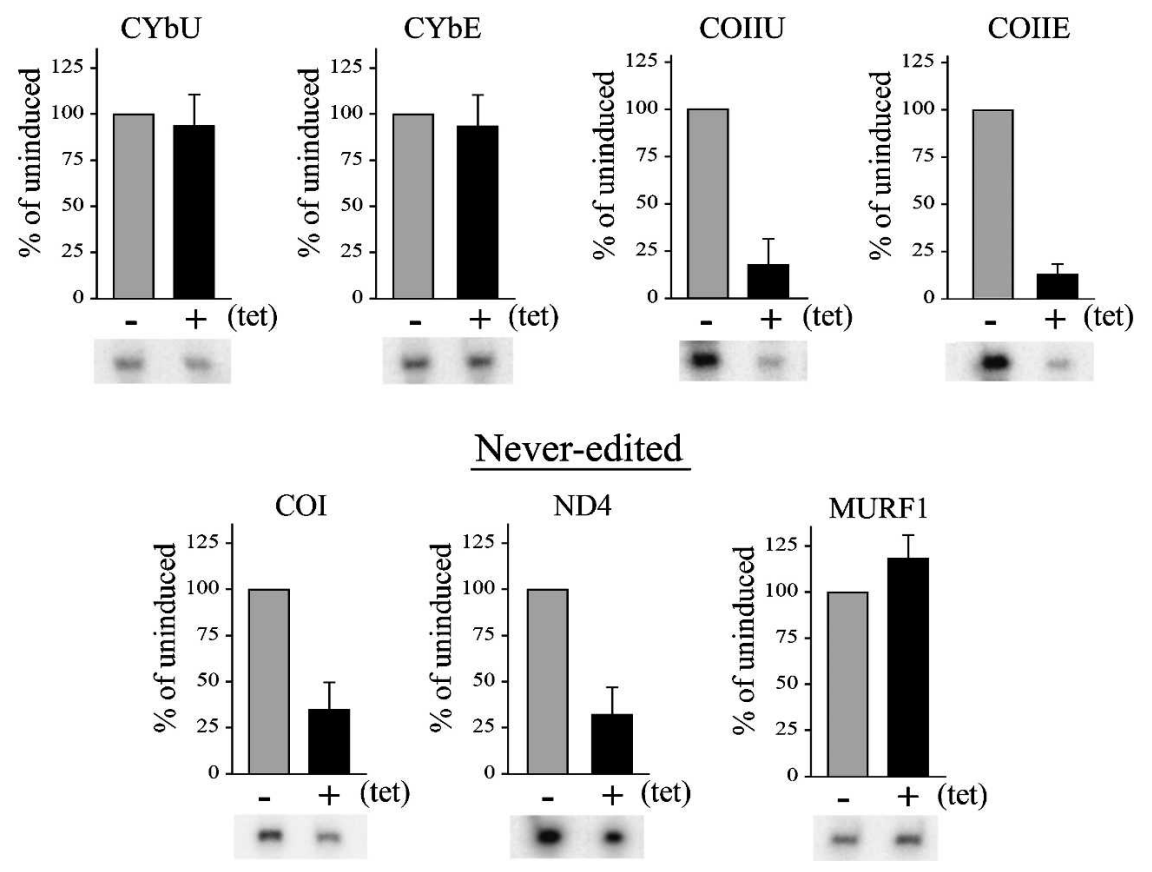

FIGURE 1. TbPRMT1 depletion decreases the stability of specific mitochondrial RNAs. Total cellular RNA was isolated from uninduced and induced ( \pm tet) TbPRMT1 RNAi cells and analyzed by poisoned primer extension using primers specific for pan-edited RNAs A6 and COIII, minimally edited RNAs CYb and COII, and the never-edited RNAs COI, ND4, and MURF1. Radiolabeled extension products were separated on an $8 \mathrm{M}$ urea/10\% acrylamide gel and visualized by PhosphorImager analysis. Relative abundance for each transcript was calculated as a percentage of the corresponding uninduced lane. Graphs represent the mean and standard deviation of three separate experiments. Representative images are shown below each graph. (U) Unedited; (E) edited.

not play a role in either the editing or stability of these pan-edited RNAs (Fig. 1). The abundance of both edited and unedited $\mathrm{CYb}$ RNAs was also unaffected (Fig. 1). However, analysis of the minimally edited COII RNA revealed a dramatic decrease in the levels of both the edited and unedited RNAs in TbPRMT1-depleted cells $(\sim 90 \%$ decrease in induced vs. uninduced cells) (Fig. 1). The corresponding decrease in both edited and unedited COII RNA levels suggests that stabilization, but not editing of this RNA, is strongly dependent on arginine methylation.
This effect could be the result of a direct destabilization of unedited COII RNA, thereby indirectly leading to decreased edited RNA due to a lack of substrate for the editing reaction. Alternatively, TbPRMT1 activity may be required to stabilize both the edited and unedited COII RNAs. We next examined the levels of never-edited RNAs in TbPRMT1 RNAi cells. MURF1 RNA levels were unaffected by TbPRMT1 depletion. In contrast, both COI and ND4 RNAs were significantly decreased when TbPRMT1 was down-regulated ( $\sim 70 \%$ decrease in induced vs. uninduced cells for both RNAs). The significant decrease in COI and ND4 RNAs upon TbPRMT1 depletion is reminiscent of the phenotype of RBP16-depleted cells (Table 1; Pelletier and Read 2003). Western blot analysis indicates that RBP16 levels are unchanged by TbPRMT1 depletion (data not shown). Thus, these data strongly suggest that methylation of RBP16 by TbPRMT1 is involved in the stabilization of the never-edited COI and ND4 RNAs.

\section{RBP16 is a substrate for TbPRMT1 in vitro}

We next wanted to determine whether RBP16 is indeed a substrate for TbPRMT1. We previously reported that TbPRMT1 was unable to methylate RBP16 in vitro (Pelletier et al. 2005), while another mitochondrial RGGcontaining protein, TBRGG1, was robustly methylated. We postulated that a cofactor such as RNA or the RBP16associated protein, p22 (Hayman et al. 2001), might be required for RBP16 methylation. In an attempt to identify conditions under which RBP16 is a substrate for TbPRMT1, we performed in vitro methylation assays as previously described (Pelletier et al. 2005) with slight modifications to the enzyme preparation (see Materials and Methods). His-RBP16 (Miller and Read 2003) was incubated with MBP-TbPRMT1 (Pelletier et al. 2005) in the presence of the methyl donor $\left[{ }^{3} \mathrm{H}\right]$ AdoMet for $18 \mathrm{~h}$ at $27^{\circ} \mathrm{C}$. As shown in Figure 2, there are two bands present in the MBP-PRMT1/His-RBP16 (lane 2), a faint band corresponding to full-length His-RBP16 (arrow) and a more prominent band that presumably corresponds to a 
TABLE 1. Effects of TbPRMT1 depletion and RBP16 depletion and overexpression on mitochondrial RNA levels

\begin{tabular}{lcccc}
\hline & RBP16 RNAi & TbPRMT1 RNAi & Myc-RBP16 WT & Myc-RBP16 TRI \\
\hline A6U & - & - & - & - \\
A6E & - & - & - & - \\
COIIU & - & $\downarrow \downarrow \downarrow$ & - & - \\
COIIE & - & $\downarrow \downarrow \downarrow$ & $\uparrow$ & - \\
COIIIU & ND & - & $\uparrow$ & - \\
COIIIE & ND & - & $\uparrow$ & - \\
CYbU & $\uparrow$ & - & - & - \\
CYbE & $\downarrow \downarrow \downarrow$ & - & - & - \\
COI & $\downarrow \downarrow$ & $\downarrow \downarrow$ & - & $\downarrow$ \\
MURF1 & ND & - & $\downarrow \downarrow$ & - \\
ND4 & $\downarrow \downarrow$ & & $\downarrow$ & - \\
\hline
\end{tabular}

Arrows indicate relative change in RNA levels for the corresponding transcript (left) in the induced cells lines indicated (top) as compared to uninduced. ( - ) No change; ( $\uparrow$ ) twofold change; $(\uparrow \uparrow)$ three- to fourfold change; $(\uparrow \uparrow \uparrow) 10$-fold change; (ND) not done.

( $\sim 14 \mathrm{Da} /$ methyl group). Such peptides will also contain an internal arginine, since trypsin does not cleave after methylated arginines (Kim et al. 1997). We previously reported that RBP16 is constitutively dimethylated on R93 and mutually exclusively methylated on R78 and R85, with R78 found to be monomethylated and R85 in di- and trimethylated forms (Pelletier et al. 2001). In the experiments presented here, RBP16 isolated from uninduced TbPRMT1 RNAi cells was modified in a manner similar to RBP16 previously observed in wild-type cells (Fig. 3A). That is, we obtained peptide masses consistent with the theoretical expected masses of unmodified peptides for nearly the entire RBP16 sequence, with

His-RBP16 degradation product (asterisk). Both of these bands are absent in the His-RBP16 alone (lane 1), demonstrating that RBP16 is a substrate for TbPRMT1 methylation activity. To determine whether a cofactor is required for the full catalytic activity of TbPRMT1 toward RBP16, we performed in vitro methylation assays in the presence of increasing amounts of p22 or an equimolar amount of the unrelated control protein, RPA32. We found that addition of $\mathrm{p} 22$ to the in vitro methylation reaction greatly stabilized full-length His-RBP16 in a concentration-dependent manner, but did not increase its methylation if the combined methylation of the two bands is considered. This effect on RBP16 stability is specific to p22, as it was not observed with an equimolar amount of RPA32. Addition of gRNA to the reaction, either in the presence or absence of p22, had no effect on either RBP16 stability or methylation (data not shown). The p22-mediated stabilization of RBP16 allows us to definitively identify RBP16 as a TbPRMT1 substrate in vitro.

\section{TbPRMT1 methylates a select subset of arginine residues within RBP16 in vivo}

Having shown that RBP16 is an in vitro substrate for TbPRMT1, we wanted to confirm that RBP16 is methylated by TbPRMT1 in vivo and to determine which of the three known methylated arginine residues within RBP16 are modified by TbPRMT1. To address these questions, we performed MALDI-TOF-MS analysis (Zhang et al. 1994) of endogenous RBP16 immunoprecipitated from mitochondrial extract of uninduced and induced TbPRMT1 RNAi cells. Immunoprecipitated RBP16 was separated by SDSPAGE and the Coomassie-stained bands were excised and subjected to tryptic digest prior to MALDI-TOF-MS. Using this method, methylated peptides can be recognized as those with masses equal to the expected mass of the unmodified peptide plus the mass of the modification the exception of the peptide containing R93. For the peptide containing R93 (Fig. 3A, peptide 11), we only obtained peptides containing dimethylated arginine, consistent with previous results (Pelletier et al. 2001). R78 and R85 containing peptides were found to be both unmethylated and methylated in the uninduced cells, also as previously reported, with the additional identification here of a monomethylated R85-containing peptide (Fig. 3A, peptides 6-10). In induced cells, in which TbPRMT1 had been depleted by RNAi, R93 was found exclusively in the dimethylated form, similar to what was observed in uninduced cells (cf. Fig. 3A, peptide 11 and Fig. 3B, peptide 6). Conversely, R78 and R85 were observed exclusively in their unmethylated forms in cells depleted for TbPRMT1 (cf. Fig. 3A, peptides 6-10 and Fig. 3B, peptide 5; note that trypsin does not cleave after unmodified arginine residues if they are followed by a proline [Coligan 1997]). These data clearly demonstrate that TbPRMT1 is responsible for

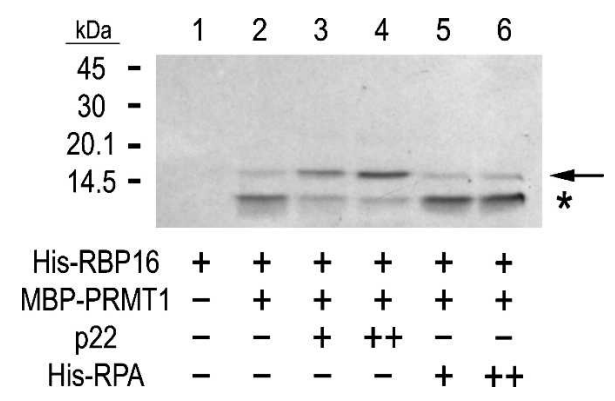

FIGURE 2. TbPRMT1 catalyzes the methylation of RBP16 in vitro. His-RBP16 was used as a substrate for in vitro methylation assays in the absence (lane 1) or presence (lanes 2-6) of recombinant MBPTbPRMT1. The RBP16-associated factor p22 (lanes 3,4) or HisRPA32 (lanes 5,6) were added at 0.15 and 0.3 molar amounts relative to RBP16. (Arrow) Full length His-RBP16; (asterisk) His-RBP16 breakdown product. 
A) Uninduced (-tet)

\begin{tabular}{|c|c|c|c|c|c|}
\hline Peptide \# & Sequence & Exp. Mass (Da) & Obs. Mass (Da) & $\Delta$ mass $(\mathrm{Da})$ & Modification \\
\hline 1 & 5 VISWMSGR & 934.469 & 934.376 & 0.093 & None \\
\hline 2 & ${ }_{13}$ GFGFIEDDADKK $_{24}$ & 1340.620 & 1340.533 & 0.087 & None \\
\hline 3 & ${ }_{25}$ QHFVHFSALQTETGGFR $_{41}$ & 1960.954 & 1960.850 & 0.104 & None \\
\hline 4 & ${ }_{42}$ ALTVGQEVEFEVASQDGR 59 & 1933.938 & 1933.821 & 0.117 & None \\
\hline 5 & ${ }_{62}$ AENVTSPGGAK $_{72}$ & 1030.516 & 1030.478 & 0.038 & None \\
\hline 6 & ${ }_{73}$ LPSGPRPPEGAGR $_{85}$ & 1289.684 & 1289.592 & 0.092 & None \\
\hline 7 & ${ }_{73}$ LPSGPRPPEGAGR $_{85}$ & 1289.684 & 1303.566 & 13.882 & Monomethyl \\
\hline 8 & ${ }_{73}$ LPSGPRPPEGAGRGR $_{87}$ & 1502.802 & 1516.585 & 13.783 & Monomethyl \\
\hline 9 & ${ }_{73}$ LPSGPRPPEGAGRGR $_{87}$ & 1502.802 & 1530.773 & 27.971 & Dimethyl \\
\hline 10 & ${ }_{73}$ LPSGPRPPEGAGRGR & 1502.802 & 1544.771 & 41.969 & Trimethyl \\
\hline 11 & ${ }_{88}$ GFGGGRGGR $_{96}$ & 819.406 & 847.266 & 27.860 & Dimethyl \\
\hline 12 & ${ }_{97}$ DFGGDR $_{102}$ & 665.177 & 664.836 & 0.341 & None \\
\hline 13 & ${ }_{107}$ GRNDNQQGGQHQSFSDDF 124 & 2035.832 & 2035.245 & 0.587 & None \\
\hline
\end{tabular}

B) Induced (+tet)

\begin{tabular}{|c|c|c|c|c|c|}
\hline Peptide \# & Sequence & Exp. Mass (Da) & Obs. Mass (Da) & $\Delta$ mass $(\mathrm{Da})$ & Modification \\
\hline 1 & ${ }_{5}$ VISWMSGR $_{12}$ & 934.469 & 934.549 & 0.080 & None \\
\hline 2 & ${ }_{13}$ GFGFIEDDADKK $_{24}$ & 1340.620 & 1340.431 & 0.189 & None \\
\hline 3 & ${ }_{25}$ QHFVHFSALQTETGGFR $_{41}$ & 1960.954 & 1960.916 & 0.038 & None \\
\hline 4 & ${ }_{42}$ ALTVGQEVEFEVASQDGR $_{59}$ & 1933.938 & 1933.807 & 0.131 & None \\
\hline 5 & ${ }_{73}$ LPSGPRPPEGAGR $_{85}$ & 1289.684 & 1289.563 & 0.121 & None \\
\hline 6 & ${ }_{88}$ GFGGGRGGR $_{96}$ & 819.406 & 847.363 & 27.957 & Dimethyl \\
\hline 7 & ${ }_{97}$ DFGGDR $_{102}$ & 665.177 & 665.311 & 0.134 & None \\
\hline 8 & ${ }_{107}$ GRNDNQQGGQHQSFSDDF $_{124}$ & 2035.832 & 2035.701 & 0.131 & None \\
\hline
\end{tabular}

FIGURE 3. TbPRMT1 methylates a select subset of arginine residues within RBP16 in vivo Endogenous RBP16 was immunoprecipitated from mitochondrial extract of uninduced $(A)$ and induced $(B)$ TbPRMT1 RNAi cells. Immunoprecipitated protein was separated by SDSPAGE, and the Coomassie-stained bands corresponding to RBP16 were excised and subjected to tryptic digest prior to MALDI-TOF-MS. (R) A methylated arginine residue.

the methylation of RBP16 at residues R78 and R85, and strongly suggest that the methylation of R93 is catalyzed by another enzyme.

\section{RBP16 arginine methylation is critical for stabilization of specific mitochondrial RNAs}

RNAi-mediated down-regulation of RBP16 results in almost complete inhibition of CYb RNA editing and an $80 \%-85 \%$ reduction in the stability of the never-edited COI and ND4 RNAs (Table 1; Pelletier and Read 2003). The TbPRMT1 depletion studies described above (Fig. 1) suggest that TbPRMT1-catalyzed RBP16 methylation may be required for the RBP16's stabilizing effect on neveredited RNAs. To directly address whether arginine methylation of RBP16 is important in the gene regulatory functions of the protein, we generated procyclic form trypanosomes that exogenously express a nonmethylatable form of RBP16 under the regulation of tet. We reasoned that if arginine methylation regulates protein:protein and/ or protein:RNA interactions important for RBP16 function, then cells expressing the nonmethylatable protein might display dominant negative phenotypes similar to those observed in RBP16 knock-down cells. A similar dominant negative approach was used to demonstrate a role for CBP methylation in transcriptional activation by estrogen receptor in mammalian cells (Chevillard-Briet et al. 2002). The Myc-RBP16-TRI cell line inducibly expresses an exogenous Myc-tagged version of RBP16 that contains $\mathrm{R} / \mathrm{K}$ mutations at the three arginine residues known to be methylated in vivo (R78, R85, and R93) (Fig. 4A). As a control, a wild-type (WT) methylatable version (Myc-RBP16-WT) was also created to determine any effects simply due to RBP16 overexpression. Stably transfected procyclic $T$. brucei cells were induced for $4 \mathrm{~d}$ in the presence of 1.0 $\mu \mathrm{g} / \mathrm{mL}$ tet prior to isolation of wholecell extract and purification of mitochondria. Western blot analysis of the cell lines expressing Myc-RBP16-WT and Myc-RBP16-TRI demonstrated that upon induction, Myc-RBP16-WT and Myc-RBP16-TRI are expressed at comparable levels and subsequently enriched in the mitochondria (Fig. 4B). Mitochondria were free of cytoplasmic contamination as shown by Western blot with antibodies against the cytoplasmic marker HSP70.4. Efficient import of Myc-RBP16-TRI into mitochondria demonstrates that arginine methylation is not required for proper subcellular localization of the protein.

We next determined whether expression of the nonmethylatable form of RBP16 has dominant negative effects on known RBP16 functions. We used poisoned primer extension to quantify RNAs that are affected by RBP16 disruption (COI, ND4, and edited and unedited $\mathrm{CYb}$ ) in cells expressing either Myc-RBP16-WT or Myc-RBP16-TRI. Total RNA was isolated from cells that had either been induced for $4 \mathrm{~d}$ in the presence of $1.0 \mu \mathrm{g} / \mathrm{mL}$ tet or left uninduced as a control. TbPRMT1-depletion studies described above (Fig. 1) indicated that protein arginine methylation impacts the stability of COI and ND4 RNAs and demonstrated that down-regulation of TbPRMT1 mimics the effect of RBP16 depletion on both COI and ND4 RNA levels. Interestingly, as shown in Figure 5, we observed no effect of Myc-RBP16-TRI expression on COI RNA levels. This may indicate that the effect observed in TbPRMT1 RNAi cells reflects the action of another methylprotein on COI RNA stability. Alternatively, methylation of RBP16 may be important for the stabilization of COI RNA, but this effect may not be manifested in a dominant 


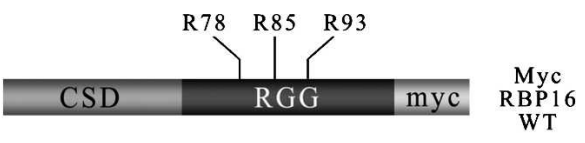

A
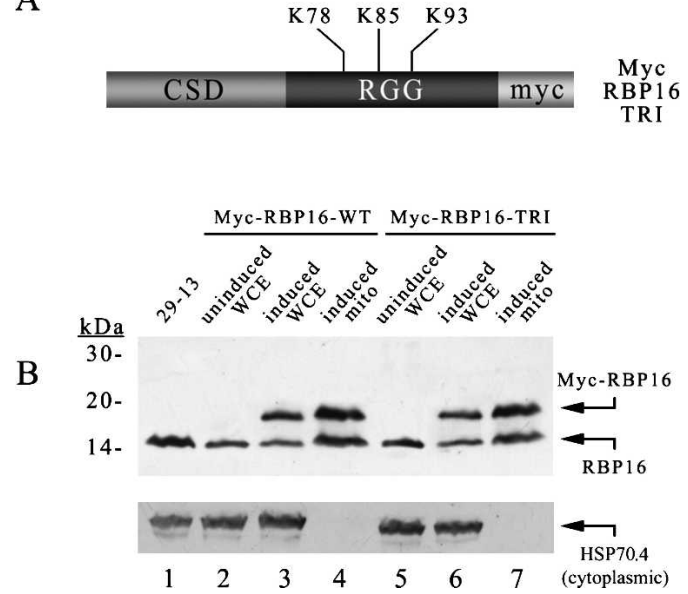

FIGURE 4. Expression of Myc-RBP16-WT and Myc-RBP16-TRI. (A) Arginine residues at positions 78, 85, and 93 of Myc-RBP16-WT (top) were mutated to lysine using site-directed mutagenesis to create the nonmethylatable triple-mutant Myc-RBP16-TRI (bottom). (CSD) Cold shock domain; (RGG) Arg-Gly rich domain; (Myc) c-Myc epitope tag. (B) Total cellular protein from 29-13 parental cells (lane 1), uninduced and induced Myc-RBP16-WT cells (lanes 2,3), uninduced and induced Myc-RBP16-TRI cells (lanes 5,6), and mitochondrial extracts from induced Myc-RBP16-WT cells (lane 4) and induced Myc-RBP16-TRI cells (lane 7) were analyzed by $\alpha$-RBP16 Western blot to verify the exogenous expression of the myc-tagged RBP16 proteins and their import into the purified mitochondria. Western blot with antibodies against the cytoplasmic marker HSP70.4 confirms that the mitochondria are essentially free of cytoplasmic contamination.

negative manner. In contrast, we did observe a striking reduction in the stability of ND4 RNA upon expression of Myc-RBP16-TRI, indicating a dominant negative effect when a nonmethylatable form of RBP16 is expressed (Fig. 5). The dominant negative effect of Myc-RBP16-TRI expression results in an $\sim 55 \%$ reduction in ND4 RNA levels as compared with $\sim 80 \%$ as seen upon RBP16 RNAi (Pelletier and Read 2003). Finally, expression of nonmethylatable Myc-RBP16-TRI had no effect on either edited or unedited CYb RNA levels (Fig. 5). These results, combined with the lack of an effect of TbPRMT1 down-regulation on CYb RNA levels (Fig. 1), suggest that methylation of RBP16 is not required for its effect on CYb RNA editing. However, while we did not observe an effect of Myc-RBP16-TRI expression on the editing or stability of CYb RNA, we unexpectedly observed a twofold stabilization of both unedited and edited $\mathrm{CYb}$ in the cells overexpressing MycRBP16-WT (Fig. 5). This stabilizing effect apparently requires methylation of RBP16, since we did not observe the same effect in the cells expressing the nonmethylatable Myc-RBP16-TRI. From these data, we conclude that arginine methylation of RBP16 is critical for its previously observed role in ND4 RNA stabilization, as well as for a newly discovered role in CYb RNA stabilization.

Because the stabilization of CYb RNA that we observed upon overexpression of Myc-RBP16-WT (Fig. 5) was a previously undefined function of RBP16, we decided to analyze the metabolism of other mitochondrial transcripts in cells overexpressing Myc-RBP16-WT and the nonmethylatable Myc-RBP16-TRI to determine the scope of these effects. Here, we examined the same subset of RNAs analyzed in TbPRMT1-depleted cells, with examples of pan-edited, minimally edited, and never-edited transcripts. Poisoned primer extension assays revealed that edited and unedited A6 and COII as well as never-edited MURF1 RNA levels were essentially unaffected by overexpression of either Myc-RBP16-WT or the nonmethylatable Myc-RBP16-TRI (Fig. 6). The slight increase in MURF1 stability in the presence of overexpressed MycRBP16-TRI does not appear to be statistically significant, as the error bars overlapped those of the Myc-RBP16-WT expression, which, in turn, did not differ significantly from uninduced controls. However, both unedited and edited COIII transcripts were stabilized in the Myc-RBP16-WT cells, similar to the effect observed for CYb RNA (cf. COIII in Fig. 6 and CYb in Fig. 5). As with CYb RNA, enhanced stabilization of COIII RNA apparently requires RBP16 methylation, as it was not observed upon overexpression of Myc-RBP16-TRI. Overall, these data suggest a more extensive role for RBP16 in the regulation of mitochondrial transcript stability than previously thought and
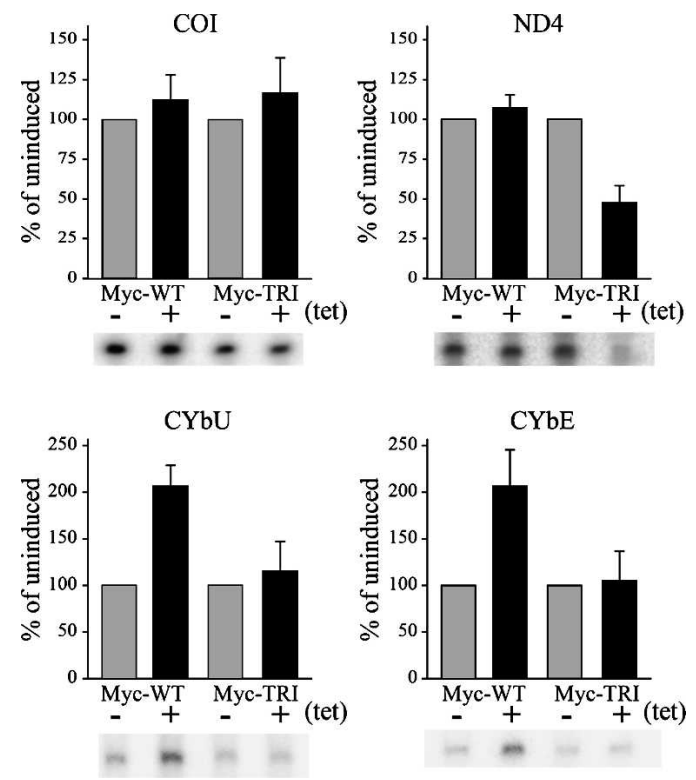

FIGURE 5. Arginine methylation of RBP16 promotes ND4 and CYb RNA stabilization. Total cellular RNA was isolated from uninduced and induced ( \pm tet) Myc-RBP16-WT and Myc-RBP16-TRI cells and analyzed by poisoned primer extension using primers specific for $\mathrm{COI}, \mathrm{ND} 4$, and CYb RNAs. Radiolabeled extension products were analyzed and the data presented as in Figure 1. (U) Unedited; (E) edited. 

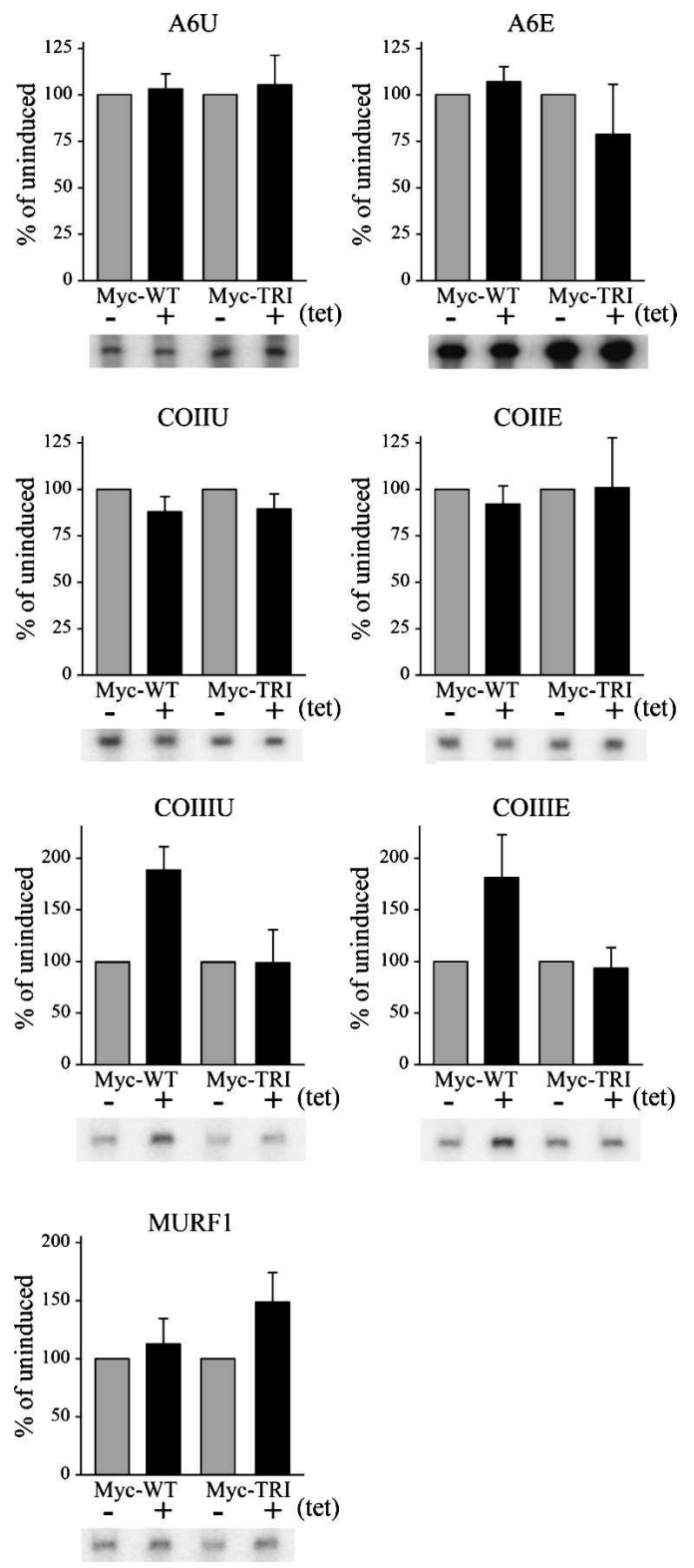

FIGURE 6. Overexpression of methylatable RBP16 promotes COIII RNA stabilization. Total cellular RNA was isolated from uninduced and induced ( \pm tet) Myc-RBP16-WT and Myc-RBP16-TRI cells and analyzed by poisoned primer extension using primers specific for A6, COII, COIII, and MURF1 RNAs. Radiolabeled extension products were analyzed and the data presented as in Figure 1. (U) Unedited; (E) edited.

implicate arginine methylation as a key modulator of this function.

\section{DISCUSSION}

Arginine methylation has been reported to affect multiple aspects of nuclear gene regulation in mammals and yeast (Bedford and Richard 2005). In this study, we asked whether gene expression in mitochondria was similarly impacted by this common post-transcriptional modification. The results in TbPRMT1-depleted cells implicate arginine methylation as a broad range regulator of mitochondrial gene expression in T. brucei. The RNA-binding protein, RBP16, is the only reported methylprotein in trypanosomes (Pelletier et al. 2001), and this protein has been shown to affect RNA stability and editing (Pelletier and Read 2003). In this study, we identified RBP16 as an effector of arginine methylation action in T. brucei mitochondria, and showed that arginine methylation is a key point of regulation for RBP16 function. We further identified effects of TbPRMT1 depletion that cannot be explained by RBP16 action, thereby implicating additional unidentified TbPRMT1 substrates in the regulation of mitochondrial gene expression.

In Table 1, we summarize the effects of RBP16 and TbPRMT1 down-regulation, as well as overexpression of wild-type and nonmethylatable RBP16 on mitochondrial RNA abundance. Given the broad RNA-binding capacity and multifunctional nature of RBP16 (Hayman and Read 1999; Pelletier et al. 2000; Pelletier and Read 2003), we postulated that the specificity of RBP16 function we observe in vivo (Pelletier and Read 2003) is likely due to regulation via interaction with transcript-specific proteins and/or by post-translational modification. We previously showed that RBP16 is post-translationally modified by methylation on multiple arginine residues in vivo and that differentially methylated forms of the protein are present within the mitochondria (Pelletier et al. 2001). Comparison of RBP16 RNAi cells and TbPRMT1 RNAi cells reveals a similar decrease in the levels of the never-edited COI and ND4 RNAs, strongly suggesting that methylation of arginine residues in RBP16 by TbPRMT1 is necessary for its RNA stabilization function. Overexpression of the nonmethylatable Myc-RBP16-TRI mimics the effects of both RBP16 and TbPRMT1 depletion on ND4 RNA levels, further supporting a role for RBP16 methylation in ND4 RNA stabilization. The absence of COI destabilization in cells overexpressing Myc-RBP16-TRI suggests that COI RNA stabilization takes place through a somewhat different mechanism than that of ND4 RNA, which is not evident as a dominant negative phenotype. Alternatively, methylation of another effector protein by TbPRMT1 may be involved in COI RNA stabilization. These studies also revealed previously unidentified roles for RBP16 in regulation of mitochondrial RNA abundance. Overexpression of wildtype RBP16 stabilized edited and unedited versions of $\mathrm{CYb}$ and COIII RNAs to similar extents. This RBP16 function also appears to be dependent on methylation, as it was not observed upon overexpression of the nonmethylatable version of the protein. The RNAs whose stability is affected by RBP16 and its methylation state do not fall into any easily recognizable pattern such as life-cycle stage specificity or eventual role of the gene products in mitochondrial physiology. However, it is notable that $\mathrm{CYb}$ and COIII 
genes are adjacent on the maxicircle, and dicistronic mRNAs containing $\mathrm{CYb}$ and COIII sequence have been reported (Koslowsky and Yahampath 1997). Since RNA editing can precede polycistronic transcript cleavage (Read et al. 1992), the similar effect of RBP16 overexpression and methylation on edited and unedited CYb and COIII RNAs could conceivably take place at the level of the precursor transcript. Thus, adjacent genomic location may sometimes be a determinant of similar modes of transcript stabilization. In contrast to the effects of RBP16 on RNA stabilization, the very dramatic effect of RBP16 down-regulation on CYb RNA editing is not recapitulated in TbPRMT1 RNAi cells. This implies that methylation of RBP16 is not important for its editing regulatory function. MALDI-TOFMS analysis showed that RBP16 from TbPRMT1 RNAi cells, while lacking methylation at $\mathrm{R} 78$ and $\mathrm{R} 85$, retains dimethylated R93 (Fig. 3; see below). Thus, we cannot rule out that R93 methylation is sufficient to maintain $\mathrm{CYb}$ editing. However, the lack of any effect of overexpression of the nonmethylatable Myc-RBP16-TRI on either edited or unedited CYb RNA levels is also consistent with the notion that methylation of RBP16 is not critical for its RNAediting-related effects. Together, these findings indicate that RBP16 methylation is important for some, but not all, aspects of RBP16 function. This supports our original hypothesis that the specificity and multifunctional nature of RBP16 are due, at least in part, to the presence of differentially modified forms of the protein.

We demonstrated that nonmethylatable RBP16 is imported into mitochondria as efficiently as endogenous RBP16 (Fig. 4). Therefore, methylation is not a prerequisite for the mitochondrial import of RBP16. This is in contrast to the well-known role of arginine methylation in regulating nucleocytoplasmic protein transport of several yeast and mammalian proteins (Bedford and Richard 2005). We do not currently know the subcellular compartment in which RBP16 undergoes methylation or whether TbPRMT1 is present in mitochondria. TbPRMT1 does not appear to have an $\mathrm{N}$-terminal sequence with similarity to trypanosome mitochondrial targeting sequences, and it is strongly predicted to be cytoplasmic by the PSORTII program. In mammalian cells, PRMT1 apparently shuttles between the nucleus and cytoplasm, with the bulk of the protein localized to the cytoplasm (Herrmann et al. 2005). To our knowledge, whether PRMTs also exist in mitochondria has not been specifically explored. Since the proteins that regulate mitochondrial gene expression in $T$. bruce $i$ are exclusively encoded in the nucleus and translated on cytoplasmic ribosomes, they could theoretically be methylated prior to mitochondrial import. However, in the absence of data regarding a potential mitochondrial localization of TbPRMT1, it remains equally plausible that at least some substrates are modified within the mitochondria. A precise determination of TbPRMT1 subcellular localization awaits the production of specific antibodies.
We showed that RBP16 is methylated by recombinant TbPRMT1 in vitro, in keeping with our results indicating that TbPRMT1-catalyzed RBP16 methylation regulates mitochondrial gene expression. We previously reported that RBP16 was either not a substrate for TbPRMT1 in vitro or that its methylation required a cofactor (Pelletier et al. 2005). Here, we show that slight modifications to the enzyme-isolation protocol yield a catalytically active preparation, such that RBP16 alone is sufficient as a substrate in the in vitro methylation reaction. Increased stabilization of RBP16 was achieved through the addition of the RBP16associated cofactor $\mathrm{p} 22$, thereby resulting in a significant increase in full-length methylated RBP16.

In wild-type $T$. brucei, RBP16 is constitutively methylated on R93, while methylation of R78 and R85 is apparently mutually exclusive (Pelletier et al. 2001). MALDI-TOF-MS analysis of RBP16 from cells depleted for TbPRMT1 revealed the absence of peptides methylated on arginines 78 or 85 . Thus, in vivo, methylation of residues $\mathrm{R} 78$ and $\mathrm{R} 85$ within $\mathrm{RBP} 16$ is catalyzed by TbPRMT1. Together with the poisoned primer extension data presented above (Fig. 1), these data define a role for methylation of specific arginine residues (R78 and R85) in the RNA stabilization function of RBP16. In contrast, R93 appears fully dimethylated in TbPRMT1-depleted cells (Fig. 3 ), indicating that this residue is methylated by another enzyme. We have identified a homolog of the type II protein arginine methyltransferase, PRMT5 (Branscombe et al. 2001; Rho et al. 2001), in the T. brucei genome. This protein, which we term TbPRMT5, possesses methyltransferase activity and is a likely candidate for catalyzing RBP16 R93 methylation (D. Pasternack and L. Read, unpubl.). We are currently examining the possibility that RBP16 serves as a substrate for both TbPRMT1 and TbPRMT5, and that together these modifications further mediate the gene regulatory effects of RBP16 (Pelletier and Read 2003).

The most dramatic consequence of TbPRMT1 downregulation is the striking reduction of COII RNA stability. Because down-regulation of RBP16 by RNAi has no effect on COII RNA levels (Table 1) (Pelletier and Read 2003), the significant destabilization of COII RNA that we observe in the TbPRMT1-depleted cells strongly argues for the existence of a methylprotein in addition to RBP16 that functions in mitochondrial gene regulation. The mitochondrial RNA-binding protein, TBRGG1, is a TbPRMT1 substrate in vitro and is therefore a potential candidate (Pelletier et al. 2005). Interestingly, we found that TBRGG1-containing complexes exhibit altered sedimentation in glycerol gradients upon TbPRMT1 depletion (C. Goulah and L. Read, unpubl.), suggesting that arginine methylation may affect TBRGG1 macromolecular interactions in vivo. Other possible mitochondrial targets for TbPRMT1 methylation include the arginine-rich MRP1 and MRP2 proteins (Vondruskova et al. 2005) as well as the RGG-motif containing TbRGGm protein, which has been identified 
in some editosome preparations, although it is not a stable editosome component (Panigrahi et al. 2003; Stuart et al. 2005). Future studies will be aimed at identification of additional mitochondrial protein arginine methylation substrates and examination of the roles of this post-translational modification in the regulation of RNA metabolism and ribonucleoprotein complex formation.

\section{MATERIALS AND METHODS}

\section{DNA oligonucleotide}

For site-directed mutagenesis, the following oligo-deoxynucleotides were used:

RBP16-R78K (5' -TCCCTCTGGTGGCTTCGGCCCTGACGG-3'); RBP16-R85K (5'-ACCGAAGCCGCGACCCTTTCCTGCTCCCTC TGG-3'); and

RBP16-R93K (5'-AAATCCCTTCCCCCTTTGCCGCCACCGAAG CC-3').

For PCR amplification of protein-coding regions, the following oligodeoxynucleotides were used (underlined bases indicate restriction sites):

RBP16myc1 (5'-GCUTCTAGAUATGATTCCGCCTTCCATAG-3'); RBP16myc2 (5'-GCUTCTAGAUAAAGTCATCGCTGAAGCTCT

$\left.\mathrm{G}-3^{\prime}\right)$;

RBP16-5'lew1 (5'-CCCUAAGCTTUATGATTCGCGCTTCCATA

GTTAAG- $\left.3^{\prime}\right)$; and

p2MYC 3' (5'-ACTAGTUGGATCCUGCAGCAGGTCTTC-3').

For poisoned primer extension analyses, the following oligodeoxynucleotides were used:

A6-3'NE (5' -GCGGATCCATTTGATCTTATTCTATAACTCC-3'); CYb-RT-GP (5'-CAACCTGACATTAAAAGAC-3'); COI-RT ( $5^{\prime}$-GTAATGAGTACGTTGTAAAACTG-3'); ND4-RT ( $5^{\prime}$-GATAAAAATATTAGTGACATTG-3'); COII-RT (5'-ATTTCATTACACCTACCAGG-3'); COIII-3' -NE (5'-GCGGATCCACTTCCTACAAACTAC- $\left.3^{\prime}\right)$; and MURF1-R (5'-CGGGATCCCAACTCAAAAACCAAGAAATTGA TAAG-3').

\section{Plasmid construction}

To create cells expressing tetracycline (tet) regulatable wild-type (WT) and mutant RBP16, we initially amplified the RBP16-coding region (Hayman and Read 1999) by 30 cycles of PCR from total procyclic form cDNA using primers RBP16mycl and RBP16myc2, which allowed introduction of XbaI restrictions sites at both ends. The PCR product was then ligated into the XbaI site of pTbmyc2 (Das et al. 1998) (a generous gift from Marilyn Parsons, SBRI) creating p2Myc-RBP16-WT. Triple-mutant Myc-RBP16 (p2MycRBP16-TRI) harboring R/K mutations at amino acids 78, 85, and 93, relative to the mitochondrial import signal cleavage, was created using the Stratagene QuikChange site-directed mutagenesis kit following the manufacturer's instructions. We utilized p2MycRBP16-WT as a template and the primers RBP16-R78K, RBP16$\mathrm{R} 85 \mathrm{~K}$, and RBP16-R93K to direct the sites of mutagenesis. To permit tet-regulated expression of Myc-tagged RBP16 proteins,
Myc-RBP16-WT and Myc-RBP16-TRI were amplified by 30 cycles of PCR from p2Myc-RBP16-WT and p2Myc-RBP16-TRI using primers RBP16-5'lew1 and p2MYC 3', which allowed introduction of HindIII and BamHI restrictions sites, respectively. The PCR products were then ligated into the HindIII and BamHI sites of pLew100 (a generous gift from George Cross, Rockefeller University) creating Myc100-RBP16-WT and Myc100-RBP16-TRI.

\section{Trypanosome growth, transfection, and induction of Myc100-RBP16}

Procyclic form T. brucei brucei clone IsTaR1 stock EATRO 164 was grown as described (Brun and Schonenberg 1979). Clonal procyclic form TbPRMT1 RNAi cells were grown as previously described (Pelletier et al. 2005). Procyclic T. brucei strain 29-13 (provided by George Cross), which contains integrated genes for T7 RNA polymerase and the tetracycline repressor, were grown in SDM-79 supplemented with $15 \%$ fetal bovine serum as described (Brun and Schonenberg 1979; Wirtz et al. 1999), in the presence of G418 $(15 \mu \mathrm{g} / \mathrm{mL})$ and hygromycin $(50 \mu \mathrm{g} / \mathrm{mL})$. For transfection, $1 \times 10^{8}$ cells were washed once in $5 \mathrm{~mL}$ of ice-cold Cytomix (van den Hoff et al. 1992) and resuspended in $0.5 \mathrm{~mL}$ of Cytomix containing $25 \mu \mathrm{g}$ of plasmid (Myc100-RBP16-WT or Myc100RBP16-TRI) linearized with NotI. Transfections (two pulses) were carried out on ice in 2-mm cuvettes using a Bio-Rad electroporator with the following settings: $800 \mathrm{~V}, 25 \mu \mathrm{F}$, and $40 \mathrm{ohms}$. Following transfection, cells were transferred into $2 \mathrm{~mL}$ of SDM79 supplemented with G418 and hygromycin and allowed to recuperate for $20 \mathrm{~h}$. Selection was then applied by the addition of $7.5 \mathrm{~mL}$ of SDM-79 medium supplemented with phleomycin $(10 \mu \mathrm{g} / \mathrm{mL}$ for a final concentration of $2.5 \mu \mathrm{g} / \mathrm{mL})$, and the cells were grown for 6 wk to obtain stable transfectants.

\section{Western blotting}

For analysis of Myc-RBP16-WT and Myc-RBP16-TRI protein levels in uninduced and induced transfectants, total cellular protein or total mitochondrial protein from $1 \times 10^{7}$ cell equivalents was separated by $17 \%$ SDS-PAGE and transferred electrophoretically to a nitrocellulose membrane (Schleicher and Schuell) at $50 \mathrm{~V}$ for $35 \mathrm{~min}$ in $10 \mathrm{mM} \mathrm{3-[Cyclohexylamino]-1-}$ propanesulfonic acid (CAPS) buffer ( $\mathrm{pH}$ 11.0) containing $10 \%$ methanol. The membrane was probed using either polyclonal anti-RBP16 antibodies (Hayman and Read 1999), anti-Myc antibodies (Covance), or anti-HSP70.4 antibodies (a generous gift from Jay Bangs, University of Wisconsin) at a 1:1000 dilution in Tris-buffered saline (TBS) with $2 \%$ dry milk. Primary antibodies were detected using goat anti-rabbit antibody coupled to horseradish peroxidase (Pierce Endogen) and detected by enhanced chemiluminescence (ECL; Amersham Pharmacia Biotech).

\section{Primer extension analyses}

Total RNA was purified from $5 \times 10^{9}$ cells of uninduced and induced Myc-RBP16-WT and Myc-RBP16-TRI-expressing cells as well as TbPRMT1 RNAi cells (Pelletier et al. 2005) (Purescript RNA Isolation Kit, Gentra Systems). Primer extensions were performed with $30 \mu \mathrm{g}$ of total RNA and 0.4 pmol of $5^{\prime}{ }^{32}$ P-labeled oligodeoxynucleotide primer. Samples were heated for $5 \mathrm{~min}$ at $95^{\circ} \mathrm{C}$, cooled on ice for $5 \mathrm{~min}$, and annealed for $60 \mathrm{~min}$ at $37^{\circ} \mathrm{C}$ in TE buffer 
(10 mM Tris- $\mathrm{HCl}$ at $\mathrm{pH} 8.0,1 \mathrm{mM}$ EDTA) containing $120 \mathrm{mM}$ $\mathrm{KCl}$. Extension reactions were performed in $50 \mathrm{mM}$ Tris- $\mathrm{HCl}(\mathrm{pH}$ 8.0), $20 \mathrm{mM} \mathrm{KCl}, 10 \mathrm{mM} \mathrm{MgCl}_{2}, 5 \mathrm{mM}$ DTT, $200 \mu \mathrm{M}$ each dATP, dCTP, and dTTP, $100 \mu \mathrm{M}$ ddGTP, $20 \mathrm{U}$ of RNaseOUT (Invitrogen), and $10 \mathrm{U}$ of AMV reverse transcriptase (Promega) for 45 $\min$ at $47^{\circ} \mathrm{C}$. RNA was removed by RNase A digestion $(2 \mu \mathrm{g})$ for $15 \mathrm{~min}$ at $37^{\circ} \mathrm{C}$ in the presence of $1 \mu \mathrm{g}$ of salmon sperm DNA, the extension products were precipitated with ammonium acetate and ethanol, and separated on a $8-\mathrm{M}$ urea/10\% polyacrylamide gel in 0.6X TBE. Gels were analyzed by overnight exposure to a Bio-Rad PhosphorImaging Screen K and scanning by Bio-Rad Personal FX with densitometry analysis in Bio-Rad Quantity One software. Each primer extension was performed at least three times, including RNA from two separate inductions.

\section{In vitro methylation assays}

MBP-TbPRMT1 was expressed and purified as described (Pelletier et al. 2005) except that cleavage of the MBP moiety was omitted, and the protein used for in vitro methylation assays was as follows. Purified His-RBP16 (4 $\mu \mathrm{g}$ ) (Miller and Read 2003) was incubated with $5 \mu \mathrm{g}$ of purified MBP-TbPRMT1 in the presence of $2 \mu \mathrm{Ci}$ $\left[{ }^{3} \mathrm{H}\right.$ ]AdoMet (GE Healthcare, $1 \mu \mathrm{Ci} / \mu \mathrm{L}, 80 \mathrm{Ci} / \mathrm{mmol}$ ) in $80 \mathrm{mM}$ Tris $(\mathrm{pH} 8.0)$ at $27^{\circ} \mathrm{C}$, in a total volume of $50 \mu \mathrm{L}$. All buffers contained $0.4 \mathrm{mM}$ of PMSF and $2 \mu \mathrm{M}$ of benzamidine. To assay for the effect of potential cofactors on the methylation of RBP16 by TbPRMT1, purified His-p22 (1.5-3.0 $\mu \mathrm{g}$ ) (Hayman et al. 2001) was added to the methylation. As a negative control, $0.5-1 \mu \mathrm{g}$ of a fragment of the papillomavirus RPA32 protein (a generous gift from Thomas Melendy, University at Buffalo), expressed in Escherichia coli with a His-tag, was added in place of p22. Reactions were carried out for $18 \mathrm{~h}$ and stopped by the addition of 5X SDS-PAGE loading buffer. Proteins were resolved by SDS-PAGE on 15\% acrylamide gels. Gels were stained and treated for fluorography as described (Pelletier et al. 2005).

\section{Immunoprecipitation experiments}

Mitochondrial vesicles from $1 \times 10^{10}$ cells of uninduced and induced TbPRMT1-RNAi cells were lysed in $0.5 \mathrm{~mL}$ of lysis buffer as described (Hayman and Read 1999). A total of $100 \mu \mathrm{g}$ of preimmune or anti-RBP16 IgG were added to $500 \mu \mathrm{L}$ of extract along with $50 \mathrm{U}$ of RNaseOUT (Invitrogen) and the sample incubated for $1 \mathrm{~h}$ at $4^{\circ} \mathrm{C}$ with gentle rocking; $100 \mu \mathrm{L}$ of washed rProtA Sepharose (Amersham) was added and the sample incubated for $1 \mathrm{~h}$ at $4^{\circ} \mathrm{C}$ with gentle rocking. Immune complexes were recovered by centrifugation at $10,000 \mathrm{~g}$ for $5 \mathrm{~min}$ and washed five times with $500 \mu \mathrm{L}$ of lysis buffer. The bound complexes were boiled in SDS sample buffer for $5 \mathrm{~min}$ and briefly pelleted prior to the supernatant being separated by SDS-PAGE. The gels were stained with Coomassie Blue and the bands corresponding to RBP16 excised.

\section{MALDI-TOF analysis}

Excised protein bands were washed in $200 \mathrm{mM}$ of $\mathrm{NHB}_{4} \mathrm{HCOB}_{3}$ containing $10 \%$ acetonitrile $(\mathrm{AcCN})$ and incubated at $37^{\circ} \mathrm{C}$ for $30 \mathrm{~min}$. The wash solution was removed and the gel plugs dried by speed-vacuum for $\sim 1 \mathrm{~h}$. One gel volume $(\sim 15 \mu \mathrm{L})$ of $20 \mathrm{mg} / \mathrm{mL}$ trypsin (Promega) in $40 \mathrm{mM} \mathrm{NHB}_{4} \mathrm{HCOB}_{3}$ containing $10 \% \mathrm{AcCN}$ was added and the sample incubated for $60 \mathrm{~min}$ at room temperature (RT). A total of $50 \mu \mathrm{L}$ of $40 \mathrm{mM} \mathrm{NHB}_{4} \mathrm{HCOB}_{3}$ containing $10 \% \mathrm{AcCN}$ was added and the sample incubated overnight at $37^{\circ} \mathrm{C}$. The supernatant was removed and the digested peptides mixture purified and concentrated using a ZipTip (Millipore) and eluted in $1 \mu \mathrm{L}$ of $0.1 \%$ trifluoroacetic acid containing $60 \%$ AcCN with saturated $\alpha$-cyano-4-hydroxycinnamic acid matrix compound (Bruker-Daltonics). Mass spectrophotometry was performed using a Bruker-Daltonics Biflex IV MALDI-TOF spectrometer and data analyzed using $\mathrm{M} / \mathrm{Z}-$ for Windows (Proteometrics, LLC).

\section{ACKNOWLEDGMENTS}

We thank Jonelle Mattiacio and Deborah Pasternack for assistance with site-directed mutagenesis and Dr. Kevin Militello for critical reading of the manuscript. We thank Dr. Marilyn Parsons for the pTbMyc2 plasmid, Dr. Thomas Melendy for His-RPA32 protein, Dr. Jay Bangs for anti-HSP70.4 antibodies, and Dr. George Cross for the 29-13 cells and the pLEW100 plasmid. This work was supported by a grant to L.K.R. from the National Institutes of Health (AI060260).

Received March 14, 2006; accepted May 8, 2006.

\section{REFERENCES}

Bedford, M.T. and Richard, S. 2005. Arginine methylation: An emerging regulator of protein function. Mol. Cell 18: 263-272.

Bedford, M.T., Frankel, A., Yaffe, M.B., Clarke, S., Leder, P., and Richard, S. 2000. Arginine methylation inhibits the binding of proline-rich ligands to Src homology 3, but not WW, domains. J. Biol. Chem. 275: 16030-16036.

Bhat, G.J., Souza, A.E., Feagin, J.E., and Stuart, K. 1992. Transcriptspecific developmental regulation of polyadenylation in Trypanosoma brucei mitochondria. Mol. Biochem. Parasitol. 52: 231-240.

Branscombe, T.L., Frankel, A., Lee, J.H., Cook, J.R., Yang, Z., Pestka, S., and Clarke, S. 2001. PRMT5 (Janus kinase-binding protein 1) catalyzes the formation of symmetric dimethylarginine residues in proteins. J. Biol. Chem. 276: 32971-32976.

Brun, R. and Schonenberg, M. 1979. Cultivation and in vitro cloning of procyclic forms of Trypanosoma brucei in a semi-defined medium. Acta Trop. 36: 289-292.

Chevillard-Briet, M., Trouche, D., and Vandel, L. 2002. Control of CBP co-activating activity by arginine methylation. EMBO J. 21: $5457-5466$

Cimato, T.R., Tang, J., Xu, Y., Guarnaccia, C., Herschman, H.R., Pongor, S., and Aletta, J.M. 2002. Nerve growth factor-mediated increases in protein methylation occur predominantly at type I arginine methylation sites and involve protein arginine methyltransferase 1. J. Neurosci. Res. 67: 435-442.

Coligan, J.E. 1997. Enzymatic digestion of proteins in solution. In Current Protocols in Protein Science (eds. J.E. Coligan et al.), Vol. 2, pp. 11.0.1-11.1.19. Wiley, Hoboken, NJ.

Côté, J., Boisvert, F.M., Boulanger, M.C., Bedford, M.T., and Richard, S. 2003. Sam68 RNA binding protein is an in vivo substrate for protein arginine N-methyltransferase 1. Mol. Biol. Cell 14: 274-287.

Das, A., Park, J.-H., Hagen, C.B., and Parsons, M. 1998. Distinct domains of a nucleolar protein mediate protein kinase binding, interaction with nucleic acids and nucleolar localization. J. Cell Sci. 111: 2615-2623.

Dreyfuss, G., Kim, V.N., and Kataoka, N. 2002. Messenger-RNAbinding proteins and the messages they carry. Nat. Rev. Mol. Cell Biol. 3: 195-205. 
Gary, J.D. and Clarke, S. 1998. RNA and protein interactions modulated by protein arginine methylation. Prog. Nucleic Acid Res. Mol. Biol. 61: 65-130.

Grams, J., McManus, M., and Hajduk, S.L. 2000. Processing of polycistronic guide RNAs is associated with RNA editing complexes in Trypanosoma brucei. EMBO J. 19T: 5525-5532.

Hayman, M.L. and Read, L.K. 1999. Trypanosoma brucei RBP16 is a mitochondrial Y-box family protein with guide RNA binding activity. J. Biol. Chem. 274: 12067-12074.

Hayman, M.L., Miller, M.M., Chandler, D.M., Goulah, C.C., and Read, L.K. 2001. The trypanosome homolog of human p32 interacts with RBP16 and stimulates its gRNA binding activity. Nucleic Acids Res. 29: 5216-5225.

Herrmann, F., Lee, J., Bedford, M.T., and Fackelmayer, F.O. 2005. Dynamics of human protein arginine methyltransferase 1 (PRMT1) in vivo. J. Biol. Chem 280: 38005-38010.

Kao, C.Y. and Read, L.K. 2005. Opposing effects of polyadenylation on the stability of edited and unedited mitochondrial RNAs in Trypanosoma brucei. Mol. Cell. Biol. 25: 1634-1644.

Kim, S., Merrill, B.M., Rajpurohit, R., Kumar, A., Stone, K.L., Papov, V.V., Schneiders, J.M., Szer, W., Wilson, S.H., Paik, W.K., et al. 1997. Identification of NPGP-methylarginine residues in human heterogeneous RNP protein Al: Phe/GlyGly-Gly-Arg-Gly-Gly-Gly/Phe is a preferred recognition motif. Biochemistry 36: 5185-5192.

Koslowsky, D.J. and Yahampath, G. 1997. Mitochondrial mRNA 3' cleavage/polyadenylation and RNA editing in Trypanosoma brucei are independent events. Mol. Biochem. Parasitol. 90: 81-94.

Li, H., Park, S., Kilburn, B., Jelinek, M.A., Henschen-Edman, A., Aswad, D.W., Stallcup, M.R., and Laird-Offringa, I.A. 2002. Lipopolysaccharide-induced methylation of $\mathrm{HuR}$, an mRNAstabilizing protein, by CARM1. J. Biol. Chem. 277: 44623-44630.

Lukes, J., Hashimi, H., and Zíková, A. 2005. Unexplained complexity of the mitochondrial genome and transcriptome in kinetoplastid flagellates. Curr. Genet. 48: 277-299.

Lukong, K.E. and Richard, S. 2004. Arginine methylation signals mRNA export. Nat. Struct. Mol. Biol. 11: 914-915.

Madison-Antenucci, S. and Hajduk, S.L. 2001. RNA editing-associated protein 1 is an RNA binding protein with specificity for pre-edited mRNA. Mol. Cell 4: 879-886.

Madison-Antenucci, S., Grams, J., and Hajduk, S.L. 2002. Editing machines: The complexities of trypanosome RNA editing. Cell 108: $435-438$.

McBride, A.E. and Silver, P.A. 2001. State of the Arg: Protein methylation at arginine comes of age. Cell 106: 5-8.

McBride, A.E., Cook, J.T., Stemmler, E.A., Rutledge, K.L., McGrath, K.A., and Rubens, J.A. 2005. Arginine methylation of yeast mRNA-binding protein $\mathrm{Npl} 3$ directly affects its function, nuclear export and intranuclear protein interactions. J. Biol. Chem. 280: 30888-30898.

Militello, K.T. and Read, L.K. 1999. Coordination of kRNA editing and polyadenylation in Trypanosoma brucei mitochondria: Complete editing is not required for long poly(A) tract addition. Nucleic Acids Res. 27: 1377-1385.

Miller, M.M. and Read, L.K. 2003. Trypanosoma brucei: Functions of RBP16 cold shock and RGG domains in macromolecular interactions. Exp. Parasitol. 105: 140-148.

Panigrahi, A.K., Allen, T.E., Stuart, K., Haynes, P.A., and Gygi, S.P. 2003. Mass spectrometric analysis of the editosome and other multiprotein complexes in Trypanosoma brucei. J. Am. Soc. Mass Spectrom. 14: 728-735.

Pelletier, M. and Read, L.K. 2003. RBP16 is a multifunctional gene regulatory protein involved in editing and stabilization of specific mitochondrial mRNAs in Trypanosoma brucei. RNA 9: 457-468.

Pelletier, M., Miller, M.M., and Read, L.K. 2000. RNA-binding properties of the mitochondrial Y-box protein RBP16. Nucleic Acids Res. 28: 1266-1275.

Pelletier, M., Xu, Y., Wang, X., Zahariev, S., Pongor, S., Aletta, J.M., and Read, L.K. 2001. Arginine methylation of a mitochondrial guide RNA binding protein from Trypanosoma brucei. Mol. Biochem. Parasitol. 118: 49-59.

Pelletier, M., Pasternack, D.A., and Read, L.K. 2005. In vivo and in vitro characterization of the major type I protein arginine methyltransferase in Trypanosoma brucei. Mol. Biochem. Parasitol. 144: 206-217.

Read, L.K., Myler, P.J., and Stuart, K. 1992. Extensive editing of both processed and preprocessed maxicircle CR6 transcripts in Trypanosoma brucei. J. Biol. Chem. 267: 1123-1128.

Rho, J., Choi, S., Seong, Y.R., Cho, W.K., Kim, S.H., and Im, D.S. 2001. Prmt5, which forms distinct homo-oligomers, is a member of the protein-arginine methyltransferase family. J. Biol. Chem. 276: 11393-11401.

Ryan, C.M., Militello, K.T., and Read, L.K. 2003. Polyadenylation regulates the stability of Trypanosoma brucei mitochondrial RNAs. J. Biol. Chem. 278: 32753-32762.

Simpson, L., Sbicego, S., and Aphasizhev, R. 2003. Uridine insertion/ deletion RNA editing in trypanosome mitochondria: A complex business. RNA 9: 265-276.

Stetler, A., Winograd, C., Sayegh, J., Cheever, A., Patton, E., Zhang, X., Clarke, S., and Ceman, S. 2005. Identification and characterization of the methyl arginines in the fragile X mental retardation protein Fmrp. Hum. Mol. Genet. 15: 87-96.

Stuart, K. and Panigrahi, A.K. 2002. RNA editing: Complexity and complications. Mol. Microbiol. 45: 591-596.

Stuart, K.D., Schnaufer, A., Ernst, N.L., and Panigrahi, A.K. 2005. Complex management: RNA editing in trypanosomes. Trends Biochem. Sci. 30: 97-105.

van den Hoff, M.J., Moorman, A.F., and Lamers, W.H. 1992. Electroporation in 'intracellular' buffer increases cell survival. Nucleic Acids Res. 20: 2902.

Vanhamme, L., Perez-Morga, D., Marchal, C., Speijer, D., Lambert, L., Geuskens, M., Alexandre, S., Ismaili, N., Göringer, U., Benne, R., et al. 1998. Trypanosoma brucei TBRGG1, a mitochondrial oligo(U)-binding protein that co-localizes with an in vitro RNA editing activity. J. Biol. Chem. 273: 21825-21833.

Vondruskova, E., van den Burg, J., Zikova, A., Ernst, N.L., Stuart, K., Benne, R., and Lukes, J. 2005. RNA interference analyses suggest a transcript-specific regulatory role for mitochondrial RNA-binding proteins MRP1 and MRP2 in RNA editing and other RNA processing in Trypanosoma brucei. J. Biol. Chem. 280: 2429-2438.

Wirtz, E., Leal, S., Ochatt, C., and Cross, G.A. 1999. A tightly regulated inducible expression system for conditional gene knock-outs and dominant-negative genetics in Trypanosoma brucei. Mol. Biochem. Parasitol. 99: 89-101.

Zhang, W., Czernik, A.J., Yungwirth, T., Aebersold, R., and Chait, B.T. 1994. Matrix-assisted laser desorption mass spectrometric peptide mapping of proteins separated by two-dimensional gel electrophoresis: Determination of phosphorylation in synapsin I. Protein Sci. 3: 677-686. 

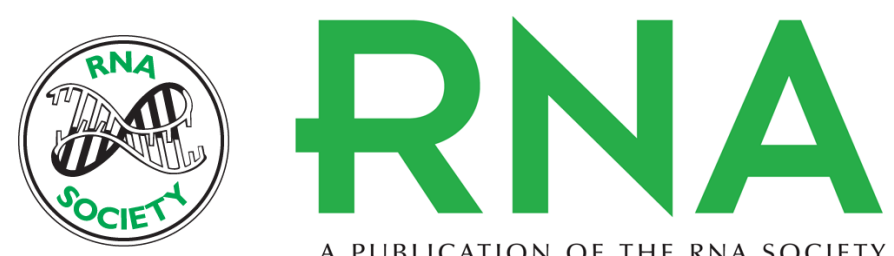

A PUBLICATION OF THE RNA SOCIETY

\section{Arginine methylation regulates mitochondrial gene expression in Trypanosoma brucei through multiple effector proteins}

Christopher C. Goulah, Michel Pelletier and Laurie K. Read

RNA 2006 12: 1545-1555

References This article cites 44 articles, 17 of which can be accessed free at:

http://rnajournal.cshlp.org/content/12/8/1545.full.html\#ref-list-1

License

Email Alerting Receive free email alerts when new articles cite this article - sign up in the box at the Service top right corner of the article or click here.

To subscribe to RNA go to:

http://rnajournal.cshlp.org/subscriptions 